\title{
Influência do zinco sobre o efeito do selênio na atividade da enzima $\delta$-ALA-D de fígado, rim e cérebro de camundongos adultos in vitro
}

NiLda Vargas Barbosa JOÃO BATISTA T. DA ROCHA

\section{RESUMO}

O selênio é um elemento traço essencial para o homem e a sua deficiência na dieta está relacionada com o desenvolvimento de uma série de patologias, como as cardiovasculares. No entanto, muitos trabalhos têm evidenciado o efeito tóxico de determinados compostos orgânicos e inorgânicos de selênio em alguns órgãos e enzimas específicas; o qual compromete o funcionamento dos mesmos. Este trabalho aborda especificamente o efeito tóxico do selênio sobre uma das enzimas envolvidas na síntese do heme a $\delta$-ALA-D - e o possível papel protetor dos íons $\mathrm{Zn}^{++}$sobre a ação tóxica deste elemento frente a enzima.

$\mathrm{O}$ composto $\left(\mathrm{CH}_{3}\right)_{2} \mathrm{C}(\mathrm{Se} \phi)\left(\mathrm{OCH}_{3}\right)$ nas concentrações testadas $(12,40$, e $120 \mu \mathrm{M})$, inibe significativamente a atividade da enzima hepática, renal e cerebral $(p<0.01)$. Também foi demonstrado que a interação do $\left(\mathrm{CH}_{3}\right)_{2} \mathrm{C}(\mathrm{Se} \phi)\left(0 \mathrm{CH}_{3}\right)$ com $\mathrm{ZnCl}(100 \mathrm{mM})$ não foi capaz de proteger a enzima da inibição provocada por selênio mas potencializou efeito inibitório do composto na $\delta$-ALA-D renal e cerebral. 


\section{ABSTRACT}

Selenium is an essential dietary trace element and its deficiency can cause some pathologies such as cardiac diseases. However, investigations have evidencied the toxicologic effect of organic and inorganic selenium compounds in the organs and enzymes. The present work examined the toxic effect of selenium on $\delta$ - ALA-D an enzyme involved in the biosynthesis of tetrapyrrol heme. A possible protective role of zinc on inhibitory effect of selenium was also evaluated.

The organic selenium compound, $\left(\mathrm{CH}_{3}\right)_{2} \mathrm{C}(\mathrm{Se} \phi)\left(0 \mathrm{CH}_{3}\right)$, caused a dose dependent inhibition of renal, hepatic and cerebral $\delta$ ALA-D $(p<0.01)$. Also was evidencied that the selenium and zinc interaction not was able to restore the $\delta$-ALA-D activity inhibited by selenium but increasing the inhibitory effect from the compound on the renal and cerebral enzyme.

\section{INTRODUÇÃO}

A $\delta$-aminolevulinato desidratase ( $\delta$-ALA-D) é uma enzima sulfidrílica que catalisa a condensação de duas moléculas do ácido delta-aminolevulínico para formar, o composto monopirrólico, porfibilinogênio (GIBSON et al.,1955). Essa reação é fundamental na biossíntese de compostos tetrapirrólicos como o grupamento heme. Consequentemente a inibição desta enzima pode prejudicar a síntese do heme, acarretando no acúmulo do ácido delta-aminolevulínico (ALA), o qual pode apresentar atividade pró-oxidante (BECHARA et.al.,1993). Como citado anteriormente a $\delta$-ALA-D, independente da fonte estudada, é uma enzima de natureza sulfidrílica (TSUKAMOTO et al.,1979, BEVAN et al.,1980) tendo portanto sua atividade inibida por uma série de agentes bloqueadores de grupos tiólicos e por metais pesados que possuam elevada afinidade por grupamentos sulfidrílicos, tais como chumbo (GOERING e FOWLER,1984,1985; GOERING et al.,1986; RODRIGUES et al., 1989,1996; GOERING,1993; ROCHA et al,1995), cobre (NELSON et al.,1981) e mercúrio (ROCHA et al.,1993, 1995).

Em mamíferos foi constatado que a $\delta$-ALA-D contém dois tipos de sítios de ligação para o $Z^{++}$, denominados sítios $A$ e $\beta$, os quais ligam $\mathrm{Zn}^{++}$com alta e baixa afinidade respectivamente (DENT et al.,1990; JAFFE,1995). O Z $\mathrm{n}^{++}$ligado ao sítio $A$ é pentacoordenado, tendo como um dos cinco ligantes um resíduo -SH de císteína, enquanto o $\mathrm{Zn}^{++}$presente no sítio $\beta$ da enzima está tetraedricamente 
coordenado a quatro ligantes de cisteína. Tem sido proposto que o papel do $\mathrm{Zn}^{++}$ligado ao sítio $A$ da $\delta$-ALA-D está diretamente relacionado com a sua atividade catalítica, enquanto o $\mathrm{Zn}^{++}$ligado ao sítio $\beta$ com a manutenção dos resíduos de cisteína no estado reduzido (JAFFE et al.,1994; EMANUELLI et.al.,1996, BEBER et.al.,1998), condição fundamental para que a enzima apresente atividade ótima.

O selênio, por sua vez é considerado um elemento traço essencial com importante papel fisiológico como componenté específico do sítio ativo de determinadas enzimas como a glutationa peroxidase (FLOHE et al.,1973; ROTRUCK et al.,1973), glutationa peroxidase de hidroperóxido de fosfolipídeos (URSINI et al.,1982, 1985,1987) e 5 deiodinase (BEHNE et al.,1990).

O mecanismo molecular pelo qual o selênio exerce sua toxicidade ainda não encontra-se estabelecido de forma definitiva; no entanto já em 1941 foi proposto que os efeitos tóxicos de determinadas formas inorgânicas de selênio como o selenito (SeO3-2) poderia estar relacionado com a oxidação de tióis, com a consequente formação de compostos intermediários (GSSeSG, $\mathrm{GSSeH}, \mathrm{H}_{2} \mathrm{Se}, \mathrm{O}^{2}$-) altamente reativos, responsáveis então pela oxidação de grupamentos sulfidrílicos (PAINTER,1941; TSEN e TAPPEL, 1958, GANTHER,1966,1968,1971; GANTHER e CORCORAN,1969; SEKO et.al.,1989; SPALLHOZ,1991). Recentemente também foi descrito que formas orgânicas de selênio também podem aumentar a taxa de oxidação de grupos - $\mathrm{SH}$ (GOEGER e GANTHER,1994; SCHONEICH et al.,1990; BARBOSA et al.,1998; MACIEL et al., 2000; JAQUES-SILVA et al.,2001). Determinados compostos orgânicos de selênio podem ser oxidados a seus respectivos selenóxidos, os quais são considerados potentes oxidantes tiólicos (FARINA et al., 2001). Várias formas orgânicas de selênio podem ainda apresentar atividade tiolperoxidase, ou seja, reduzir $0 \mathrm{H}_{2} \mathrm{O}_{2}$ à $\mathrm{H}_{2} \mathrm{O}$ na presença de substratos tiólicos tais como a GSH (COTGREAVE et al.,1992).

Com relação aos efeitos do selênio na atividade da $\delta$ ALA-D, foi observado que a exposição a algumas formas inorgânicas de selênio causa um efeito inibitório na enzima proveniente de embriões de ratos in vivo. De acordo com esses resultados, evidências mostram que compostos orgânicos e inorgânicos de selênio inibem a atividade da $\delta$-ALA-D de ratos in vitro (BARBOSA et al., 1998; JAQUES- SILVA et al., 2001, MACIEL et al.,2000; FARINA et al., 2001). Todavia, um possivel papel protetor do $\mathrm{Zn}^{++}$ainda não foi investigado de forma detalhada.

O objetivo do presente estudo foi investigar o possível Ciência \& Natura, Santa Maria, 24: 49 - 62, 2002. 51 
papel protetor do zinco sobre o efeito inibitório do selênio frente a atividade da enzima de fígado, cérebro e rim de camundongos in vitro.

\section{MATERIAIS E MÉTODOS: \\ Animais experimentais:}

Foram utilizados camundongos adultos provenientes do biotério central da Universidade Federal de Santa Maria. Os animais foram mantidos em uma sala com temperatura controlada $\left(22-25^{\circ} \mathrm{C}\right)$, iluminação natural e alimentação (Guabi-RS) e água ad libitum.

\section{Preparação Tecidual:}

Os animais foram anestesiados com éter e mortos por decapitação. Os órgãos, cérebro, rim e fígado, foram rapidamente removidos, pesados e colocados no gelo e então homogeneizados em $5,7 e 10$ volumes de $\mathrm{NaCl}(150 \mathrm{mM})$. O homogeneizado foi centrifugado a $4000 \mathrm{~g}$, a $4^{\circ} \mathrm{C}$, por $10 \mathrm{~min}$. O sobrenadante obtido (S1) foi utilizado para determinação da atividade da $\delta$-ALA-D.

\section{Determinação da atividade da $\delta$-ALA-D:}

$A$ atividade da $\delta$-ALA-D de camundongos foi determinada de acordo com o método de SASSA (1982), pela quantidade de produto formado (porfibilinogênio). O meio de incubação, cujo volume final era de $250 \mathrm{ml}$, continha $84 \mathrm{mM}$ de tampão fosfato de potássio, $\mathrm{pH} 6.4,2.5 \mathrm{mM}$ de ALA e $100 \mathrm{ml}$ de preparação tecidual. A quantidade de proteína incubada nos ensaios variou de 0.4 - $0.5 \mathrm{mg}$ para cérebro, e de 0.8 - $1.1 \mathrm{mg}$ para rim e fígado.

A reação foi iniciada pela adição de $100 \mathrm{ml}$ da preparação tecidual a um meio contendo o substrato (ALA). As amostras foram incubadas por $1-4$ horas a $39^{\circ} \mathrm{C}$. A reação foi interrompida pela adição de $150 \mathrm{ml}$ de TCA $10 \%$ contendo $0.01 \mathrm{M}$ de $\mathrm{HgCl}_{2}$. O produto da reação foi determinado com o reagente de Ehrlich, a $555 \mathrm{~nm}$. O coeficiente de absorção molar para o produto de reação do porfibilinogênio com o reagente de Ehrlich é de $6,1 \times 10^{4}$. A reação foi linear com o tempo de incubação e com a quantidade de proteína em todas as condições experimentais. 


\section{Determinação de proteínas:}

A determinação do conteúdo protéico das amostras foi efetuada de acordo com o método de BRADFORD (1976), utilizandose albumina de soro bovino como padrão.

\section{RESULTADOS:}

Papel do zinco sobre o efeito do 2-fenilseleno-2-metóxipropano na atividade da $\delta$-ALA-D hepática, renal e cerebral de camundongos in vitro

Sabe-se que determinadas formas orgânicas de selênio inibem significativamente a atividade da enzima $\delta$-ALA-D de cérebro, fígado e rim de ratos adultos in vitro (BARBOSA et al.,1998) e que existem evidências na literatura atribuindo ao $\mathrm{Zn}^{++}$um papel direto na função catalítica da $\delta$-ALA-D ou ainda corroborando a hipótese de que o mesmo estabiliza a forma ativa da enzima possivelmente por impedir a oxidação de grupos -SH essenciais à $\delta$-ALA-D. Com base nesses dados investigou-se o efeito concomitante do $\mathrm{ZnCl}_{2}(100 \mathrm{mM})$ e do composto orgânico, 2-fenilseleno-2-metóxi-propano (12, 40 e $120 \mu \mathrm{M})$, sobre a atividade da enzima; afim de determinar se o efeito inibitório do mesmo sobre a atividade da $\delta$-ALA-D poderia estar relacionado com a remoção de íons $\mathrm{Zn}^{++}$do seu sítio ativo.

Os resultados obtidos (Figuras 1, 2 e 3 ) mostram que 0 composto de selênio testado causa uma inibição concentração-dependente $(12,40$ e $120 \mu \mathrm{M})$ na atividade da enzima dos respectivos tecidos utilizados $(p<0.001)$.

Também evidencia-se que o zinco $(100 \mathrm{mM})$ além de não proteger a $\delta$-ALA-D da inibição provocada por selênio (Figuras 1,2 , e 3) ainda apresenta um efeito inibitório per se sobre a enzima de origem hepática (Figura 1).

A reação foi iniciada pela adição do sobrenadante hepático (S1) a um meio contendo $84 \mathrm{mM}$ de tampão fosfato de potássio, $\mathrm{pH} 6.4,2,5 \mathrm{mM}$ de $\mathrm{ALA}, \mathrm{ZnCl}_{2}(100 \mathrm{mM})$ e as concentrações de selênio indicadas. $\mathrm{O}$, Controle; $\bullet, \mathrm{ZnCl}_{2}$.

Esses resultados (Figuras 2 e 3 ) mostram ainda que a inibição causada pelo composto orgânico de selênio, 2-fenilseleno2-metóxi-propano, na $\delta$-ALA-D de rim e cérebro foi potencializada pela adição de $\mathrm{ZnCl}_{2}(100 \mathrm{mM})$ ao meio de incubação $(p<0.01)$. No entanto, para a enzima hepática esse efeito da interação selênio + zinco aumentando a inibição promovida pelo composto de selênio não foi significativo $(p=0.98)$. 
Fígado

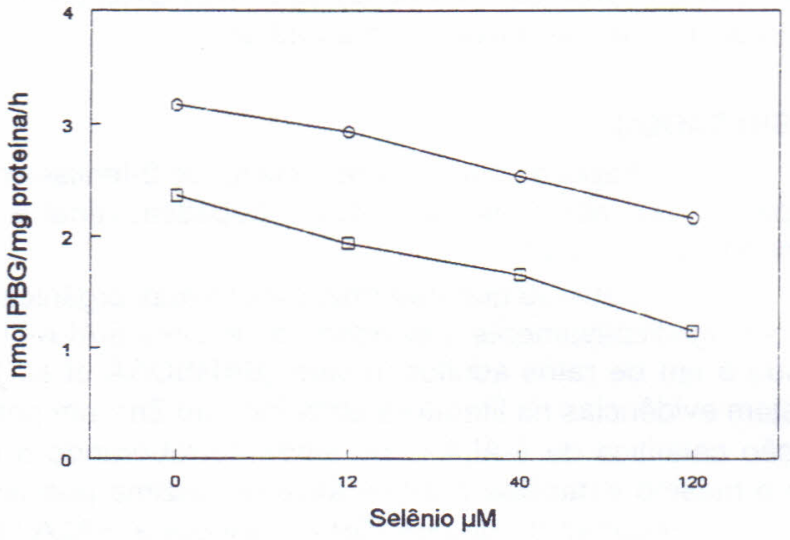

Fig. 1. Efeito do $\mathrm{ZnCl} 2$ e do $\left(\mathrm{CH}_{3}\right)_{2} \mathrm{C}(\mathrm{Se} \phi)\left(\mathrm{OCH}_{3}\right)$ na atividade da $\delta$-ALA-D hepática de camundongos in vitro. Os dados são expressos como média $\pm E P(n=5)$.

$\operatorname{Rim}$

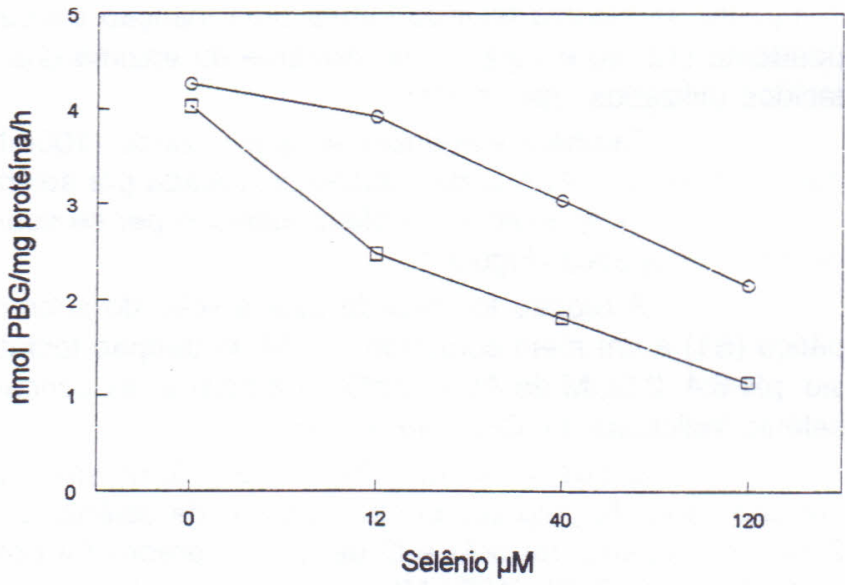

Fig. 2. Efeito do $\mathrm{ZnCl}$ e do $\left(\mathrm{CH}_{3}\right)_{2} \mathrm{C}(\mathrm{Se} \phi)(\mathrm{OCH} 3)$ na atividade da $\delta$-ALA$D$ renal de camundongos in vitro. Os dados são expressos como média $\pm E P(n=5)$. 
A reação foi iniciada pela adição do sobrenadante renal (S1) a um meio contendo $84 \mathrm{mM}$ de tampão fosfato de potássio, $\mathrm{pH}$ $6.4,2,5 \mathrm{mM}$ de ALA , $\mathrm{ZnCl}_{2}(100 \mathrm{mM})$ e as concentrações de selênio indicadas. $\mathrm{O}$, Controle; , $\mathrm{ZnCl}_{2}$.

Cérebro

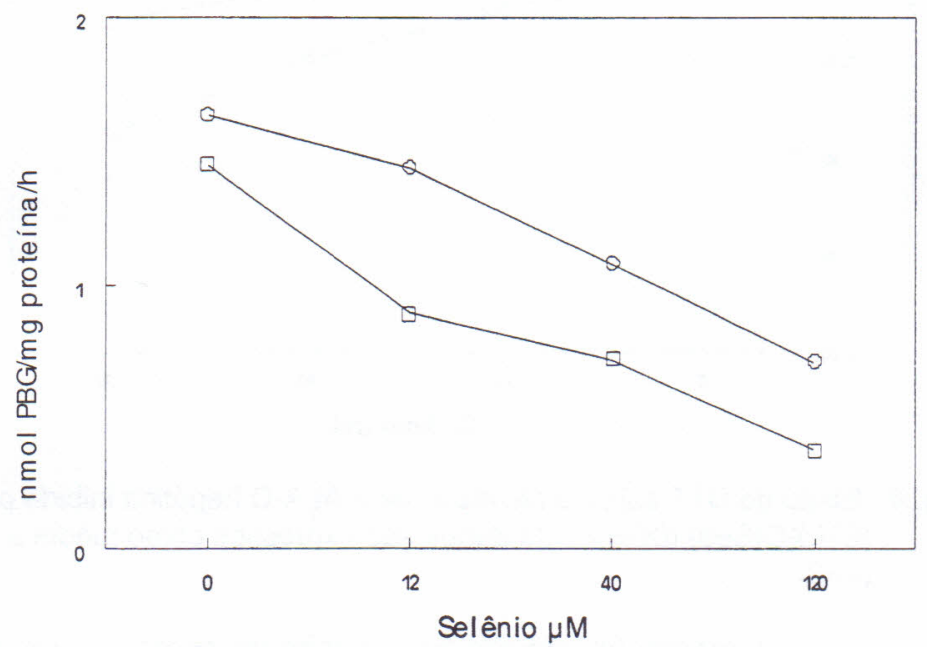

Fig. 3. Efeito do $\mathrm{ZnCl}_{2}$ e do $\left(\mathrm{CH}_{3}\right)_{2} \mathrm{C}(\mathrm{Se} \phi)\left(\mathrm{OCH}_{3}\right)$ na atividade da $\delta$-ALA-D cerebral de camundongos in vitro. Os dados são expressos como média $\pm \operatorname{EP}(n=6)$.

A reação foi iniciada pela adição do sobrenadante cerebral (S1) a um meio contendo $84 \mathrm{mM}$ de tampão fosfato de potássio, $\mathrm{pH} 6.4,2,5 \mathrm{mM}$ de $\mathrm{ALA}, \mathrm{ZnCl}_{2}(100 \mathrm{mM})$ e as concentrações de selênio indicadas. $\mathrm{O}$, Controle; , $\mathrm{ZnCl}_{2}$.

Uma vez que a ação inibitória do 2-fenilseleno-2-metóxipropano sobre a atividade da $\delta$-ALA-D parece não envolver a perda de zinco do sítio ativo da enzima, procurou-se identificar o possível mecanismo pelo qual esses compostos estariam inibindo a enzima. Para isso testou-se o papel do DTT, um agente redutor de grupamentos sulfidrílicos, sobre a atividade da $\delta$-ALA-D inibida por selênio . A Figura 4 revela que a adição de DTT $(10 \mathrm{mM})$ ao meio de incubação, restaura completamente a atividade da $\delta$-ALA-D hepática de camundongos. 
Fígado

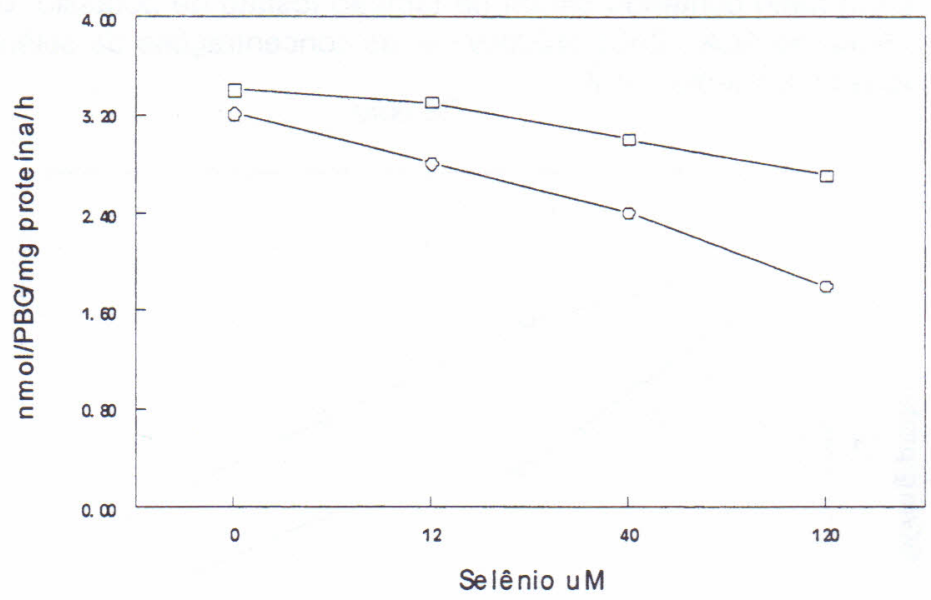

Fig. 4. Efeito do DTT sobre a atividade da $\delta$-ALA-D hepática inibida pelo $\left(\mathrm{CH}_{3}\right)_{2} \mathrm{C}(\mathrm{Se} \phi)\left(\mathrm{OCH}_{3}\right)$. Os dados são expressos como média $\pm \mathrm{EP}$ $(n=3)$.

A reação foi iniciada pela adição do sobrenadante hepático (S1) a um meio contendo $84 \mathrm{mM}$ de tampão fosfato de potássio, $\mathrm{pH} 6.4,2,5 \mathrm{mM}$ de ALA , DTT (10mM) e as concentrações de selênio indicadas. $\bullet$, Controle; O, DTT.

Resultados similares foram observados para a enzima renal e cerebral (dados não mostrados). Esses resultados indicam que o mecanismo pelo qual o selênio inibe a d-ALA-D possivelmente envolva a oxidação de resíduos cisteinil presentes no sítio $A$ ou $B$ da enzima.

\section{DISCUSSÃO:}

Os resultados exibidos no presente estudo demonstram que a enzima $\delta$-ALA-D de fígado, rim e cérebro de camundongos pode ser considerada um alvo em potencial a ação inibitória do composto de selênio testado. Já havia sido descrito na literatura que algumas formas de selênio inibem significativamente a atividade da $\delta$-ALA-D de ratos adultos in vitro (BARBOSA et al.,1998;MACIEL et al.,2000; FARINA et al.,2001), assim como metais pesados que apre- 
sentam grande afinidade por grupos -SH de biomoléculas endógenas como cobre (NELSON et al.,1981) mercúrio (ROCHA et al.,1993, 1995; EMANUELLI et al.,1998) cádmio (RODRIGUES et al.,1989) e chumbo (GOERING e FOWLER,1984,1985; GOERING et al.,1986; RODRIGUES et al., 1989,1996; GOERING,1993; ROCHA et al,1995). De acordo com tais estudos nesse trabalho evidencia-se que o composto, 2-fenilseleno-2-metóxi-propano, apresenta também um efeito inibitório significativo sobre a atividade da enzima $\delta$-ALA-D de camundongos nos diferentes tecidos estudados.

Sabe-se que após a remoção de íons zinco da enzima os grupos -SH da mesma são facilmente oxidados, situação que pode ocasionar a perda da atividade enzimática. Recentemente foi demonstrado que este $\mathrm{Zn}^{++}$tem função estrutural de evitar a oxidação de resíduos cisteinil do sítio $\beta$ da enzima (BEBER et al.,1998; EMANUELLI et al.,1998) e que o BAL, um composto tiólico, inibe a $\delta$-ALA-D por quelar os íons zinco presentes neste sítio (EMANUELLI et al.,1996).

Um resultado interessante obtido neste estudo foi com relação ao papel do zinco no efeito inibitório do selênio. Uma vez que estudos apontam para o zinco uma função antioxidante, era de se esperar que a adição de $\mathrm{ZnCl}_{2}$ no ensaio pudesse proteger a atividade da enzima inibida por selênio ou ainda não interferir no efeito inibitório do composto. Porém constatou-se que a presença de zinco $(100 \mathrm{mM})$ não protegeu a $\delta$-ALA-D do efeito inibitório do selênio e sim potencializou a ação inibitória do composto sobre a atividade da enzima renal e cerebral. Esses dados sugerem que no ensaio enzimático possivelmente tenha ocorrido a formação de um complexo $\left(\mathrm{ZnCl}_{2}+\right.$ Selênio) com uma afinidade maior por grupamentos -SH.

Para $\delta$-ALA-D hepática observou-se ainda que o zinco sozinho inibe a enzima.

Dentro deste contexto fica evidente que mecanismo pelo qual o composto de selênio testado inibe a enzima $\delta$-ALA-D possivelmente envolva um outro mecanismo que não a remoção de íons $\mathrm{Zn}^{++}$presentes no sítio $\mathrm{B}$ da enzima; uma vez que o $\mathrm{ZnCl}_{2}$ não foi capaz de recuperar a atividade da mesma.

Uma característica importante $\delta$-ALA-D é a essencialidade de seus grupos - $\mathrm{SH}$ no estado reduzido para desempenhar sua ação catalítica. A utilização de $10 \mathrm{mM}$ de DTT, um agente redutor, no ensaio enzimático foi capaz de abolir totalmente a inibição causada pelos três compostos testados. Esse antagonismo ocorre provavelmente pela interação dos grupos -SH do próprio DTT 
com os compostos presentes no meio de incubação, evitando assim a oxidação de grupos -SH essenciais da enzima e permitindo que mesma desempenhe sua função catalítica normal. Isto demonstra que o selênio inibe a $\delta$-ALA-D por causar uma oxidação reversível de grupos -SH presentes no sítio ativo da enzima e não por remover íons zinco ligados a esses grupamentos sulfidrílicos.

As concentrações do composto de selênio que inibiu a $\delta$-ALA-D in vitro relatadas neste trabalho está dentro da faixa de concentrações de selênio encontradas no sangue de pessoas expostas a altos níveis desse elemento (GREGER e LANE,1987). Consequentemente, quando extrapolado para uma situação in vivo é razoável supor que o mecanismo pelo qual o selênio produz toxicidade, esteja em parte, relacionado com a inibição da $\delta$-ALA-D e com a biossíntese do heme.

\section{REFERÊNCIAS BIBLIOGRÁFICAS}

Barbosa, N.B.V.; Rocha, J.B.T.; Zeni, G.; Emanuelli, T.; Beque, M.C. \& Braga, A L. (1998). Effect of organic forms selenium on daminolevulinate dehydratase from liver, kidney and brain of adult rats. Toxicology Applied Pharmacology. 49, 243-253.

Beber, F A; Wolsmeister,J; Brigo, M.J.K; Silva, M.C.J; Pereira, C.N. and Rocha, J.B.T. (1998). Ascorbic acid inhibition of hepatic deltaaminolevulinate dehydratase is mediated by na oxyding system present in liver supernatants. Int. J. Vitam. Nutr. Res. (In press).

Behne D.;Kyriakopoulos, A (1990). Indentification of type I iodothyronine $5^{\circ}$-deiodinase as a selenoenzyme. Biochem. Biophys. Res. Commun. 173, 1143-1149.

Bechara E.J.H; Medeiros, M.H.G.; Monteiro, H.P.; Lima, H.; Pereira, M.; Demasi, B.; Costa, M.; Abdall, C. A; Onuki, J.; Wendel, C.M.A and Masci, P.D. (1993). A free radical hypothesis of lead poisoning and inborn porphyrias associated with 5 -aminolevulinic acid overload. Química Nova. 16, 385-392.

Bevan, D.R.; Bodlaender, P. and Shemin. D. (1980)Mechanism of porphobilinogen synthase. Requirement of $\mathrm{Zn2}+$ for enzyme activity. Journal of Biological Chemistry. 255, 2030-2035.

Cotgreave, I.A ; Moudeus, P.; Brattsand, R.; Hallberg, A ; Andersson, C.M.; Engman, L. (192). a (phenylselenyl) acetophenone 
derivatives with glutathione peroxidase: Like activity. A comparisom with Ebselen. Biochem. Pharmacol. 4443, 793-802.

Dent, A J.; Beyersmann, D.; Block, C. and Hasnain, S.S. (1990).

Two different zinc sites in bovine 5-aminolevulinate dehydratase distinguished by exyend $\mathrm{X}$-ray absorption fine structure. Biochemistry. 29, 7822-7828.

Emanuelli, T.; Rocha, J.B.T.; Pereira, M. E.; Porciúncula, L.O and Souza, D. (1996). Effects of mercuric chloride intoxication and 2,3-dimercaptopropanol (BAL) treatment on delta-aminolevulinate dehydratase from brain, kidney and liver of adult mice. Pharmacology \& Toxicology. 79, 136-143.

Emanuelli, T.; Rocha, J.B.T.; Pereira, M.E.; Nascimento, P.C.; Souza, D.O G \& Beber, F.A (1998). Delta-aminolevulinate dehydratase inhibition by 2,3- dimercaptopropanol is mediated by chelation of zinc from a site involved in maintaining cysteynil residues in reduced state. Pharmacology and Toxicology. 83, 95-103.

Farina, M.; Folmer, V.; Bolzan, R.; Andrade, L.;Zeni, G.; Braga, A and Rocha, J.B.T. (2001). Selenoxides inhibit d-aminolevulinic acid dehydratase. Toxicology Letters. 119, 27-37.

Flohe, L.; Gunzler, W. A; Schock, H.H. (1973). Glutathione peroxidase: A selenium enzyme. FEBS Lett. 32, 132-134.

Ganther, H.E. (1971). Reduction of the selenotrisulfide derivative of glutathione to a persulfide analog by glutathione reductase. Biochemistry. 10, 4089-4098.

Ganther, H.E. (1968). Selenotrisulfides. Formation by reaction of thiols with selenious acid. Biochemistry. 7, 2898-2905.

Ganther, H.E. (1966). Enzimic synthesis of dimethyl

selenide from sodium selenite in mouse extract. Biochemistry. 10, 4089-4098.

Ganther, H.E.; Corcoran,C. (1969). Selenotrisulfide. II. Cross-linking of reduced pancreatic ribonuclease with selenium. Biochemistry. 8, 2557-2563.

Gibson, K.D.; Neuberger, A ; Scott, J.J. (1955). The purification and properties of delta-aminolevulinic acid dehydratase. Biochem. J. 61, 618-629.

Goering, P.L. (1993). Lead protein interactions as a basis for lead toxicity. Neurotoxicology. 14, 45-60.

Goering, P.L. and Fowler, B.A (1984). Regulation of lead inhibition of d-aminolevulinic acid dehydratase by a low molecular weigth, high affinity renal lead-binding protein. J. 
Pharmacol. Exp. Therap. 14, 45-60.

Goering, P.L.; Fowler, B.A (1986). Mechanism of renal lead-binding protein reversal of d-aminolevulinic acid dehydratase inhibition by lead. Pharmacol. Exp. Therap. 237, 220-225.

Goering, P.L.; Mistry, P. and Fowler, B.A (1986). A low molecular weight lead-binding protein in brain attenuates lead inhibition of delta-aminolevulinic acid dehydratase. Comparison with a renal lead-binding protein. J. Phamacol. Exp. Therap. 237, 220-225.

Greger, J.L. and Lane, H.W. (1987). The toxicology of dietary tin, aluminium and selenium. Nutritional Toxicology. (J.N.Hathcock, ed). Vol.2. Academic. Press, San Diego, C.A.

Jacques-SilvaM.C.; Nogueira, C.W.; Broch, L.C.; Flores, E.E.M \& Rocha, J.B.T. (2000). Diphenyl diselenide and ascorbic acidchange deposition of selenium and ascorbic acid in liver and brain of mices. Pharmacology \& Toxicology. In Press.

Jaffe, E.K. (1995). Porphobilinogen synthase, the first source of heme's asymmetry. J. Bioenergetics and Biomembranes. 27, no 2.

Linder, M.C. (1990). Nutrition and metabolism of the trace elements. 7, 216-276.

Maciel, E.N.; Bolzan, R.; Braga, A L and Rocha, J.B.T. (2000). Diphenyl diselenide and diphenyl ditelluride differentially affect $d$ aminolevulinate dehydratase from liver, kidney and brain of mice. Journal of Biochemical and Molecular Toxicology, 14,1-10.

Nelson, H.M.; Ughes, M. A ; Meredith, P.A (1981). Zinc, cooper and delta-aminolevulinic acid dehydratase in vitro. Toxicology, 21,261266.

Painter, E.P. (1941). The chemistry and toxicity of selenium compounds which special reference to the selenium problem. Chem. Rev. 28, 179-213.

Rocha, J.B.T.; Freitas, A J.; Marquez,, M.B.; Pereira, M. E.; Emanuelli, T \& Souza, D.O (1993). Effects of methylmercury exposure during the second stage of rapid postnatal brain growth on delta-aminolevulinate dehydratase (E.C. 4.2.1.24) of suckling rats. Brazilian Journal of Medical and Biological Research. 26, 1077-1083.

Rocha, J.B.T.; Pereira, M.E.; Emanuelli, T.; Christofari, R.S. \& Souza, D. O (1995). Effeccts of mercury chloride and lead acetate treatment during the secound stage of rapid postnatal brain growth on ALA-D activity in brain, liver, kidney and blood of suckling 
rats. Toxicology. 100, 27-37.

Rodrigues, A L.; Bellinaso, M. L. and Dick, T. (1989). Effect of some metals ions on blood and liver delta-aminolevulinate dehydratase of Pimelodus malacatus (pisces, pimelodidae). Comp. Biochem. Physiol. 94B, 65-69.

Rodrigues, A L.; Rocha, J.B.T.; pereira, M.E. \& Souza, D.O (1996). Delta-aminolevulinic acid dehydratase activity in weanling and adult rats exposed to lead acetate. Bull. Environ. Contam. Toxicol. 57, 47-53.

Rotruck, J.T.; Pope, A L.; Ganther, H.E.; Swanson, A B.; Hafeman, D.G.; Hoestra, W.G. (1973). Selenium: Biochemical role as a component of glutathione peroxidase. Science. 179, 558-560.

Sassa, S. (1982). Delta- aminolevulinic acid dehydratase assay. Enzyme. 28,133-145.

Seko,Y.; satio, Y.; Kitahara, J.; Imura, N. (1989). Active oxygen generation by the reaction of selenite with reduced glutathione in vitro. In: Wendel, A (ed). Selenium in biology and medicine. Springer, Berlin Heidelberg. New York. pp:70-73.

Spallhoz, J.E. (1994). On the nature of selenium toxicity and carcinostatic activity. Free Radical Biology \& Medicine. 17,45-64.

Tsen, C.C., Tappel, A L. (1958). Catalytic oxidation of glutathione and other sulphydril compounds biselenite. J. Bio. Chem. 233, 1230-1232.

Tsukamoto, I.; Youshinaga, T.; and Sano, S. (1979). The role of zinc with special reference to the essential thiol groups in deltaaminolevulinic acid dehydratase of bovine liver. Biochimica et Biophysica Acta. 570, 167-178.

Ursini, F.; Maorino, M.; Valente, M.; Ferri, K.; Gregolin, C. (1982). Purification of pig liver of a protein which protects lipossomes and biomembranes from peroxidadtive degradation and exhibits glutathione peroxidase activity on phosphatidylcholine hydroperoxidase. Biochem. Biophys. Acta. 710,197-211.

Ursini, F.; Maiorino, M and Grregolin, Carlo. (1985). The selenoenzyme phospholipid hydroperoxide glutathione peroxidase. Biochimica. Et. Biophysica. Acta. 839, 62-70.

Ursini, F and Bindoli, A (1987). The role of selenium peroxidases in the protection against oxidative damage of membranes. Chemistry and Physics of Lipids. 44, 255-276. 
Nilda Vargas Barbosa JOÃO BATISTA T. DA ROCHA Departamento de Química - CCNE Universidade Federal de Santa Maria Santa Maria, RS - Brasil 